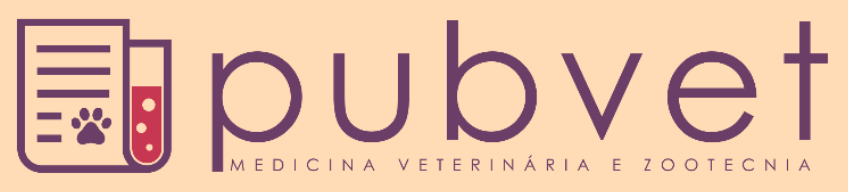

https://doi.org/10.31533/pubvet.v14n7a607.1-6

\title{
Bloqueio do nervo femoral por abordagem pré-íliaca e do tronco lombossacral por abordagem parassacral em ovino: relato de caso
}

\author{
Juliano Nunes de Oliveira ${ }^{1} \bullet$, David Ronald Travagin ${ }^{2} \bullet$, Andreza Furtado de $\operatorname{Souza}^{1 \oplus}$, Marina \\ Bassi Soares Jacinto $^{3 \bullet}$, Juan Claudio Nunes de Oliveira ${ }^{4}$, Fabricio de Oliveira Frazilio ${ }^{5}$ \\ ${ }^{I}$ Residente no Hospital Veterinário da Faculdade de Medicina Veterinária e Zootecnia/FAMEZ da Universidade Federal do Mato Grosso do \\ Sul, Departamento de Anestesiologia Veterinária e Emergência. Campo Grande-MS Brasil. \\ ${ }^{2}$ Medico Veterinário da FAMEZ da Universidade Federal do Mato Grosso do Sul, Departamento de Anestesiologia Veterinária e Emergência. Campo Grande-MS \\ ${ }^{3}$ Aluna da Faculdade de Medicina Veterinária e Zootecnia/FAMEZ da Universidade Federal do Mato Grosso do Sul. Campo Grande-MS Brasil. \\ ${ }^{4}$ Aluno da Faculdade Vale do Aço, Departamento de Medicina Veterinária. Açailândia-MA Brasil. \\ ${ }^{5}$ Professor Adjunto da Faculdade de Medicina Veterinária e Zootecnia/FAMEZ da Universidade Federal do Mato Grosso do Sul, \\ Departamento de Anestesiologia Veterinária e Emergência. Campo Grande, MS Brasil. E-mail: \\ *Autor para correspondência, E-mail: juliano_arena@hotmail.com
}

Resumo. Técnicas anestésicas e analgésicas, locais e regionais, são uteis em situações onde se é contraindicada a anestesia geral e epidural. As técnicas locorregionais são consideradas incremento para anestesia geral, uma vez que promovem uma analgesia eficiente no transoperatório, gerando uma diminuição na dose utilizada do anestésico geral, e no resgate analgésico no pós-cirúrgico. Esse relato tem como objetivo apresentar uma nova abordagem ao nervo femoral e ao nervo isquiático ou tronco lombossacro devido à altura do bloqueio, visando procedimentos mais altos no membro, impossibilidade da epidural e evitar os efeitos adversos da epidural. Além de pequenos ruminantes terem a capacidade de acordar da anestesia e se levantar por serem menos pesados. O procedimento anestésico/cirúrgico duro quase 3 horas e o paciente se manteve estável todo o tempo, sem alterações nos parâmetros vitais, e no pós-operatorio imediato, o animal já estava de pé e se alimentando. Animal foi mantido em plano superficial na anestesia, com reflexo palpebral presente e globo ocular rotacionado. Como foi utilizado vaporizador universal não foi registrada a concentração alveolar mínima a qual a anestesia foi mantida. A abordagem pré-iliaca do nervo femoral e a abordagem parasacral do tronco lombosacro é uma técnica de anestesia regional com analgésica eficiente e de fácil execução desde que guiado por um localizador de nervos periféricos. É uma técnica que reduz volume e consequentemente custo anestésico, pode ser usada quando não é possível realizar anestesia geral, diminui a necessidade de anestésico geral, não tem os riscos da anestesia epidural, sendo uma ótima escolha na contra indicação ou erro na técnica de epidural.

Palavras chave: anestesia locorregional, bloqueio perineural, neurolocalizador, pequenos ruminantes

\section{Femoral nerve block by pre-iliac approach and lumbosacral trunk by parasacral approach in sheep: case report}

Abstract. Anesthetic and analgesic techniques, local and regional, are useful in situations where general and epidural anesthesia is contraindicated. Locoregional techniques are considered an increment for general anesthesia, since they promote efficient analgesia during the operation, generating a decrease in the dose used of the general anesthetic, and in the analgesic rescue in the postoperative period. This report aims to present a new approach to the femoral nerve and the sciatic nerve or lumbosacral trunk due to the height of the block, aiming at higher procedures on the limb, impossibility of the epidural and avoiding the adverse effects of the epidural. In addition to small ruminants having the 
ability to wake up from anesthesia and get up because they are less heavy. The anesthetic / surgical procedure lasted almost 3 hours and the patient remained stable all the time, with no changes in vital parameters, and in the immediate postoperative period, the animal was already standing and eating. Animal was kept in a superficial plane during anesthesia, with eyelid reflex present and rotated eyeball. As a universal vaporizer was used, the minimum alveolar concentration at which anesthesia was maintained was not recorded. The pre-iliac approach to the femoral nerve and the parasacral approach to the lumbosacral trunk is a technique of regional anesthesia with efficient analgesics and easy to perform as long as guided by a peripheral nerve locator. It is a technique that reduces volume and consequently anesthetic cost, can be used when general anesthesia is not possible, reduces the need for general anesthetic, does not have the risks of epidural anesthesia, being a great choice in the contraindication or error in the epidural technique.

Keywords: locoregional anesthesia, perineural block, neurolocator, small ruminants

\section{Bloqueo del nervio femoral con abordaje pre-ilíaco y tronco lumbosacro con abordaje parasacral en ovejas: reporte de un caso}

Resumen. Las técnicas anestésicas y analgésicas, locales y regionales, son útiles en situaciones en las que la anestesia general y epidural está contraindicada. Las técnicas locorregionales se consideran un incremento para la anestesia general, ya que promueven una analgesia eficiente durante la operación, generando una disminución en la dosis utilizada de la anestesia general y en el rescate analgésico en el postoperatorio. Este informe tiene como objetivo presentar un nuevo enfoque para el nervio femoral y el nervio ciático o el tronco lumbosacro debido a la altura del bloqueo, con el objetivo de procedimientos más altos en la extremidad, imposibilidad de la epidural y evitar los efectos adversos de la epidural. Además de los pequeños rumiantes que tienen la capacidad de despertarse de la anestesia y levantarse porque son menos pesados. El procedimiento anestésico / quirúrgico duró casi 3 horas y el paciente permaneció estable todo el tiempo, sin cambios en los parámetros vitales, y en el período postoperatorio inmediato, el animal ya estaba de pie y comiendo. El animal se mantuvo en un plano superficial durante la anestesia, con reflejo del párpado presente y globo ocular girado. Como se utilizó un vaporizador universal, no se registró la concentración alveolar mínima a la que se mantuvo la anestesia. El abordaje pre-ilíaco del nervio femoral y el abordaje parasacro del tronco lumbosacro es una técnica de anestesia regional con analgésicos eficientes y fácil de realizar siempre que esté guiado por un localizador de nervio periférico. Es una técnica que reduce el volumen y, en consecuencia, el costo de la anestesia, se puede utilizar cuando la anestesia general no es posible, reduce la necesidad de anestesia general, no tiene los riesgos de la anestesia epidural, siendo una gran opción en la contraindicación o error en la técnica epidural.

Palabras clave: anestesia locorregional, bloqueo perineural, neurolocalizador, pequeños rumiantes

\section{Introdução}

Fraturas em ruminantes ocorrem com relativa frequência, sendo oriundas muitas vezes de brigas nos rebanhos, durante a monta, trauma em uma distocia ou manuseio e pisoteio pela mãe (Mulon, 2013). Essas lesões levam a perdas econômicas significativas para a cadeia produtiva (Spadeto Junior et al., 2011).

Técnicas anestésicas e analgésicas, locais e regionais, são uteis em situações onde se é contraindicada a anestesia geral e epidural. As técnicas loco regionais são consideradas incremento para anestesia geral, uma vez que promovem uma analgesia eficiente no transoperatório, gerando uma diminuição na dose utilizada do anestésico geral, e no resgate analgésico no pós-cirúrgico (Grimm et al., 2015; Klaumann \& Otero, 2013; Portela et al., 2013).

Uma alternativa à anestesia epidural para gerar analgesia do membro pélvico é o bloqueio dos nervos femoral e ciático, visto que, esses são uteis em casos em que não seja necessário o bloqueio bilateral dos membros pélvicos e quando a anestesia espinhal for contra indicada (Campoy et al., 2010; Mahler \& 
Adogwa, 2008; Portela et al., 2013), sendo que os nervos ciático e femoral são os principais feixes nervosos oriundos do tronco lombossacro e do plexo lombar, respectivamente (Gurney \& Leece, 2014; Otero \& Portela, 2018). O nervo femoral na abordagem lateral pré-iliaca encontra se dentro do compartimento psoas. Ele é oriundo dos nervos L4, L5 e L6, seguindo em sentido ventrolateral, cercado pelo músculo psoas maior e o músculo psoas menor. O ponto de realização desse bloqueio é lateral à vertebra lombar L5, ventral à crista ilíaca (Otero \& Portela, 2018; Silva et al., 1994).

Os ramos de L6, S1 e S2, dão origem ao tronco lombo sacral que forma o nervo isquiático. O Troco Lombo sacral faz um percurso intrapélvico e repousa sobre a fáscia pélvica na região média de corpo do ílio, sendo nessa região cercada pelo músculo sacro caudal pela região ventrolateral em direção medial, e pelo músculo elevador em direção lateral, ele é sobreposto pelos músculos glúteo médio, superficial e piriforme, onde se torna o nervo isquiático e segue até o forame isquiático maior. O ponto de realização desse bloqueio é no início do segundo terço da distância entre a margem dorsal do ílio e a tuberosidade isquiática (Otero \& Portela, 2018).

Esse relato tem como objetivo apresentar a abordagem ao nervo femoral e ao nervo isquiático ou tronco lombo sacro em um ovino, devido à altura do bloqueio, visando procedimentos mais proximais ao membro na impossibilidade da epidural ou evitar os efeitos adversos da epidural, além de permitir que o animal consiga se manter em estação.

\section{Relato de caso}

Foi encaminhado ao setor de clínica médica e cirúrgica de grandes animais da Faculdade de Medicina Veterinária e Zootecnia da Universidade Federal do Mato Grosso do Sul - FAMEZ/ UFMS um ovino, fêmea de aproximadamente 6 meses, sem raça definida, $21 \mathrm{~kg}$ pertencente ao rebanho da própria instituição. O animal foi atendido no dia 14 de janeiro de 2020, tendo como queixa principal claudicação.

$\mathrm{O}$ animal foi encontrado no pasto, afastado do rebanho e claudicava ao andar. No exame físico apresentou apatia, 120 de frequência cardíaca (FC), 70 de frequência respiratória (FR), temperatura $\left(\mathrm{T}^{\circ}\right)$ 39,9 , pulso das artérias digitais forte, mucosa normocorada, tpc 2 e 1 movimento ruminal em $3 \mathrm{~min}$. Foi realizado exame de imagem e na radiografia constatou-se fratura completa simples em espiral, em terço distal da diáfise de fêmur direito, onde se optou pela abordagem cirúrgica de osteossíntese.

Após jejum alimentar prévio de 12 horas e hídrico de 8 horas, foi conduzido ao setor de cirurgia de grandes animais para realizar osteossíntese distal de fêmur. Na anestesia foi realizado a MPA com $20 \mu \mathrm{g} / \mathrm{kg}$ de detomidina e $0,2 \mathrm{mg} / \mathrm{kg}$ de morfina por via intravenosa, após $40 \mathrm{~min}$ foi aplicado cetamina $2,5 \mathrm{mg} / \mathrm{kg}$ e diazepam $0,2 \mathrm{mg} / \mathrm{kg}$. O animal foi sondado com uma sonda endotraqueal 10 e mantido na anestesia inalatória com isoflurano diluído em $60 \%$ oxigênio, infusão intravenosa de detomidina $10 \mu \mathrm{g} / \mathrm{kg} / \mathrm{h}$ e mantido na ventilação mecânica.

Os parâmetros vitais do animal: FC, FR, capnografia (ETCO2), temperatura e pressão artéria invasiva foram monitorados por um monitor multi paramétrico. Foi feito a tricotomia e antissepsia do membro para cirurgia e para a realização de bloqueio locorregional. Primeiramente foi realizada a abordagem lateral pré-iliaca do nervo femoral. Segundo foi realizado o bloqueio do tronco lombossacral por abordagem parassacral. Técnicas essas guiadas por neuro estimulação.

A abordagem do nervo femoral pré-iliaco guiado por neuroestimulção (Figura 1) ocorreu com a introdução da agulha unipolar isolada na pele paralelo L5 por volta de $5 \mathrm{~cm}$ com angulação caudomedial em direção ao trocânter, ventral a crista ilíaca. $\mathrm{O}$ eletrodo positivo estava posicionado na altura da articulação do joelho, aparelho estava configurada a $2 \mathrm{~mA}, 1000 \mu \mathrm{Seg}$ e $2 \mathrm{~Hz}$, ao primeiro estimulo nervoso, diminui para 1,5mA e aprofundou a agulha, diminuindo gradativamente a amperagem até uma resposta muscular entre 0,5 e $0,3 \mathrm{~mA}$. Foi injetado $4 \mathrm{ml}$ de uma composição com $2 \mathrm{ml}$ de lidocaína $2 \%$ e $2 \mathrm{ml}$ de ropivacaina $1 \%$, que em seguida parou a resposta muscular ao neuro estimulador. A resposta muscular esperada pela abordagem pré-iliaca do nervo femoral é a contração do musculo quadríceps femoral, ocasionando extensão da articulação do joelho. Deve ser constata a ausência de estimulo em $0,2 \mathrm{~mA}$ e a falta de resistência durante a injeção.

A abordagem parassacral do tronco lombossacral guiada por neuroestimulção (Figura 2) se dá com a introdução perpendicular ao plano cutâneo da agulha unipolar no início do terço médio de uma linha 
traçada entre a margem dorsal da crista ilíaca e a tuberosidade isquiática (Otero \& Portela, 2018). Com o eletrodo positivo na altura do joelho, manteve se a mesma calibração do bloqueio anterior e aprofundou-se a agulha até a resposta muscular desejada, diminuindo a corrente gradativamente até uma resposta entre 0,5 e 0,3mA. Foi constatado a ausência de estimulo em $0,2 \mathrm{mAe}$ a falta de resistência durante a injeção e injetado $4 \mathrm{ml}$ da mesma solução do bloqueio anterior. A resposta desejada para a realização do bloqueio do tronco lombossacral por abordagem parassacral é a contração do musculo semimembranoso e semitendinoso, com flexão da articulação do joelho, contração dos músculos grastrocnêmios, extensão do tarso ou musculo tibial e flexão do tarso. Devido à grande quantidade de ramos nervoso no troco lombossacral, pode haver respostas musculares variadas.

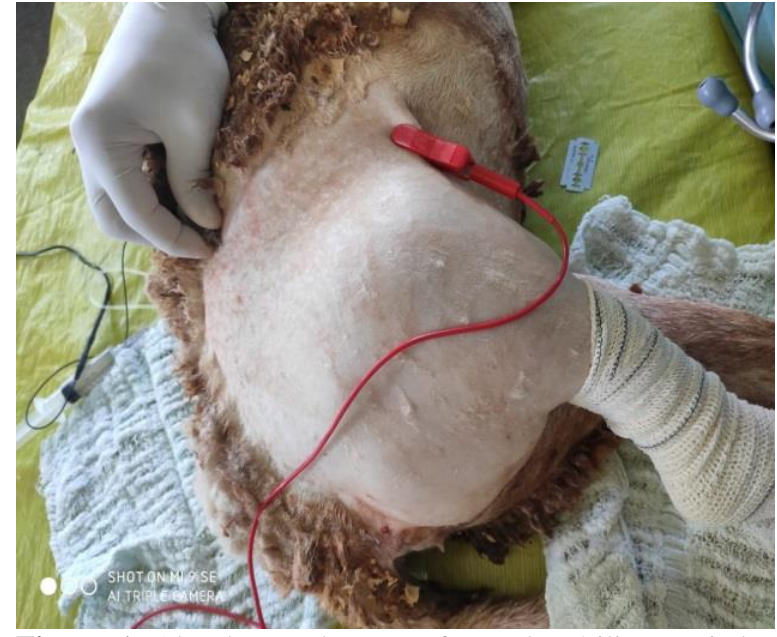

Figura 1. Abordagem do nervo femoral pré-iliaco guiado por neuroestimulção.

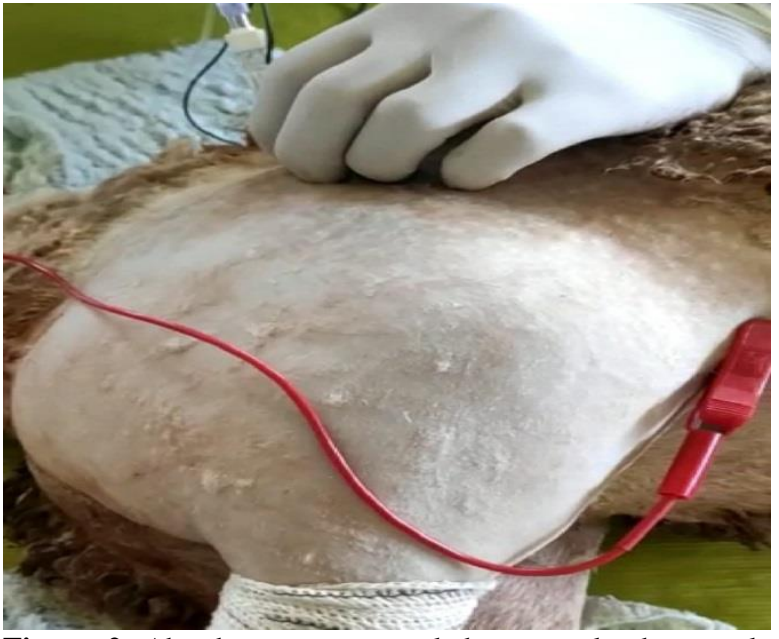

Figura 2. Abordagem parassacral do tronco lombossacral guiada por neuroestimulação.

O procedimento anestésico/cirúrgico duro quase 3 horas e o paciente se manteve estável todo o tempo, sem alterações nos parâmetros vitais, e no pós-operatório imediato, o animal já estava de pé e se alimentando (Figura 3). A pressão arterial média (PAM) se manteve por volta de $80 \mathrm{mmHg}$ por todo o procedimento, frequência cardíaca se manteve por volta de 120, frequência respiratória entre 20 e 30 movimentos por minuto. $\mathrm{O}$ animal foi mantido em plano superficial na anestesia, com reflexo palpebral presente e globo ocular rotacionado. Como foi utilizado vaporizador universal não foi registrado a concentração alveolar mínima (CAM) a qual a anestesia foi mantida.

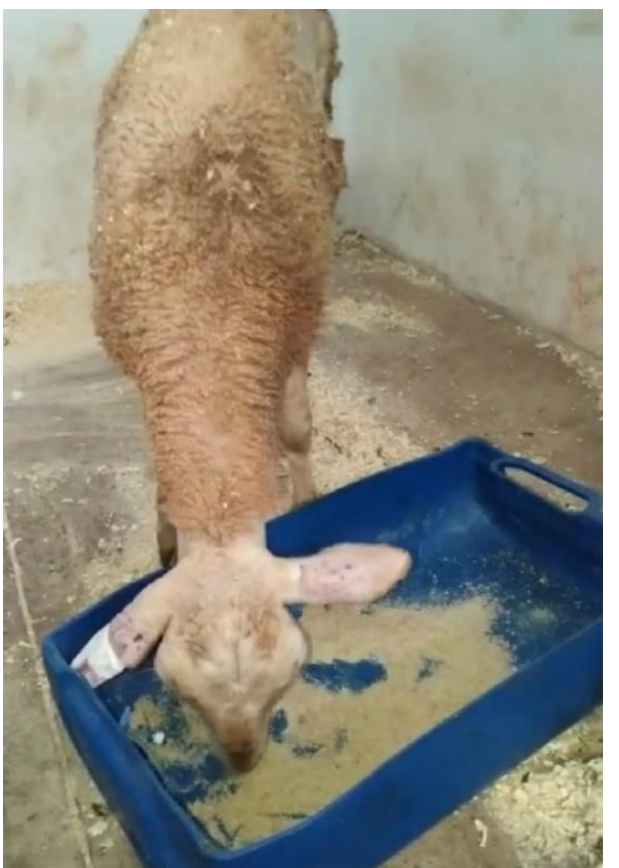

Figura 3. Pós-operatório imediato animal já estava de pé e se alimentando. 


\section{Discussão}

Apesar da presença abundante desses animais na maioria das regiões do país, servindo até mesmo em modelos experimentais, encontra-se pouca ou nenhuma literatura relacionada à anestesia de membros em pequenos ruminantes. Em pequenos animais a anestesia locorregional é bastante difundida, com abordagens diversas para o mesmo nervo, sendo muito utilizada para evitar o bloqueio bilateral dos membros pélvicos, impossibilidade de realização da epidural ou contra indicação da mesma. Otero \& Portela (2018) descrevem os bloqueios do membro pélvico como ótima alternativa analgésica, para trans e pós-cirúrgico, sendo essas hoje muito praticadas em pequenos animais. Cabala (2016) relata a utilização de bupivacaina no bloqueio do isquiático e femoral para cirurgias ortopédicas em cães com duração de 10 horas, sendo adequada também para o pós-operatório. Cabala (2016) utilizou neurolocalizador para realização de vários bloqueios em cães e bovinos, entre eles o isquiático e femoral, demonstrando assim a eficiência na técnica guiada por localizador de nervos periféricos, além da redução do volume e consequentemente dos custos.

O único trabalho encontrado de anestesia regional do nervo isquiático e femoral em ovinos é o de Silva et al. (1994), que realizou um experimento com o bloqueio do isquiático e femoral de 30 ovinos, e concluiu que a técnica é eficiente e de fácil execução, podendo ser usada na ausência de anestesia geral ou epidural. $\mathrm{O}$ mesmo usou a abordagem inguinal do nervo femoral, cranial ao músculo pectíneo e a abordagem lateral proximal do nervo isquiático, caudal ao musculo vasto lateral e ao trocânter maior do fêmur. Essa técnica pode ser feita às cegas, pois as referências anatômicas são faces de serem localizadas e não possui nenhuma estrutura importante com risco de perfuração ou lesão a não ser o próprio nervo e a artéria femoral que é uma das referências. Diferente da técnica usada por Silva et al. (1994) a técnica usada nesse relato deve ser guiada por neuro localizador ou ultrassom devido à profundidade muscular e risco de perfuração de órgãos ou cavidade abdominal.

A abordagem pré-iliaca do nervo femoral e a abordagem para sacral do tronco lombo sacral, é a técnica usada nesse relato, tendo como vantagens a dessensibilização de todo o membro pélvico, permitindo assim procedimentos em regiões proximais. Essa técnica permite dessensibilização no trans e pós-operatório sem bloqueio bilateral, permitindo que assim que recuperado da anestesia o paciente possa se locomover, mesmo que só em três membros. Quanto menor o animal mais fácil a locomoção nessa situação. A técnica guiada por neuro localizador permite maior precisão na execução do bloqueio e redução do volume anestésico e custos. Otero \& Portela (2018) descrevem a utilização a associação desses dois bloqueios em cães para regiões de quadril, coxa, joelho, e todo o membro distalmente e até mesmo para amputação. A técnica usada por Silva et al. (1994) não tem alcance tão proximal quanto a descrita nesse relato, não sendo recomendada para procedimentos como amputação ou a nível de quadril e fêmur proximal.

A técnica de bloqueio de neuro eixo é a mais utilizada em pequenos ruminantes, porém nem sempre é possível aplica-la. Grimm et al. (2015) citam em cães como contra indicações da epidural, pacientes com distúrbios hemorrágicos, pacientes hipovolêmicos ou hipotensos, pois pode se agravar a hipotensão. Animais com infecções de pele também possuem contra indicação, pois pode ser uma fonte de contaminação da via epidural. Otero \& Portela (2018) descrevem em cães como efeitos adversos a hipotensão, apneia, laceração de sacrodural, lesão iatrogênica ou contaminação, prurido ou reação alérgica. (Grimm et al., 2015) relatam efeitos adversos semelhantes em ruminantes. Mota (2017) também descreve esses afeitos adversos, e relata que os riscos na execução do bloqueio do isquiático e femoral e muito baixo e o efeito analgésico e comparável ao da epidural sendo assim uma escolha eficiente em substituição à técnica de epidural. A abordagem pré-íliaca e parassacral realizada neste artigo possuem o risco de perfuração de peritônio, alças intestinais, baço, rins e vasos sanguíneo abdominais de grande calibre. Porém esses riscos são eliminados com a técnica guiada por neuro localizador como foi utilizada neste artigo ou guiada por ultrassonografia. Além das taxas de erro da técnica ser quase nula.

Carneiro Silva et al. (1994) citam as contraindicações da anestesia geral ou dissociativa como arritmias, sensibilidade de miocárdio e hipotensão aumentando os riscos ao paciente, porém a técnica do bloqueio do isquiático e femoral não necessitou de anestesia geral anulando esses riscos. Neste relato foi utilizada anestesia geral inalatória com isoflurano devido à abordagem aqui realizada ser dificultosa 
com o animal acordado, além do procedimento cirúrgico realizado ser complexo e necessitava de total imobilidade do paciente, com isso optou-se pela anestesia geral inalatória. Porém a analgesia gerada pelo bloqueio permitiu manter o animal imóvel e estável em plano superficial durante todo o procedimento, diminuindo assim os riscos da anestesia geral.

\section{Considerações finais}

A abordagem pré-iliaca do nervo femoral e a abordagem parasacral do tronco lombosacro é uma técnica de anestesia regional com analgésica eficiente e de fácil execução desde que guiado por um localizador de nervos periféricos. É uma técnica que reduz volume e consequentemente custo anestésico. Pode ser usada quando não é possível realizar anestesia geral, diminui a necessidade de anestésico geral, não tem os riscos da anestesia epidural, sendo uma ótima escolha na contra indicação ou erro na técnica de epidural.

\section{Referências bibliográficas}

Cabala, R. W. (2016). Uso de anestesia locorregional periférica em caninos e bovinos. Um estudo clínico e experimental. Tese (Doutorado). Escola de Medicina Veterinária da Universidade Federal de Minas Gerais. Plataforma Sucupira. Belo Horizonte. MG. BR.

Campoy, L., Bezuidenhout, A. J., Gleed, R. D., Martin-Flores, M., Raw, R. M., Santare, C. L., Jay, A. R., \& Wang, A. L. (2010). Ultrasound-guided approach for axillary brachial plexus, femoral nerve, and sciatic nerve blocks in dogs. Veterinary Anaesthesia and Analgesia, 37(2), 144-153. DOI: https://doi.org/10.1111/j.1467-2995.2009.00518.x

Grimm, K., Lamont, L., Tranquilli, W., Greene, S., \& Robertson, S. (2015). Anestesiologia e analgesia em veterinária. Editora Roca.

Gurney, M. A., \& Leece, E. A. (2014). Analgesia for pelvic limb surgery. A review of peripheral nerve blocks and the extradural technique. Veterinary Anaesthesia and Analgesia, 41(5), 445-458. DOI: https://doi.org/10.1111/vaa.12184

Klaumann, P. R., \& Otero, P. E. (2013). Anestesia locorregional em pequenos animais. Roca.

Mahler, S. P., \& Adogwa, A. O. (2008). Anatomical and experimental studies of brachial plexus, sciatic, and femoral nerve-location using peripheral nerve stimulation in the dog. Veterinary Anaesthesia and Analgesia, 35(1), 80-89. DOI: https://doi.org/10.1111/j.1467-2995.2007.00356.x

Mota, T. M. (2017). Estudo comparativo entre o bloqueio combinado dos nervos femoral e ciático e a anestesia epidural lombossacra, em coelhos (Oryctolagus Cuniculus) submetidos à osteossíntese femoral.

Mulon, P.-Y. (2013). Management of long bone fractures in cattle. In Practice, 35(5), 265-271. DOI: https://doi.org/10.1136/inp.f2869

Otero, O. E., \& Portela, D. A. (2018). Anestesia regional em animais de estimação. Editora MedVet.

Portela, D. A., Otero, P. E., Briganti, A., Romano, M., Corletto, F., \& Breghi, G. (2013). Femoral nerve block: a novel psoas compartment lateral pre-iliac approach in dogs. Veterinary Anaesthesia and Analgesia, 40(2), 194-204. DOI: https://doi.org/10.1111/j.1467-2995.2012.00765.x

Silva, F. O. C., Pereira, C. C. H., \& Bombonato, P. P. (1994). Anestesia do membro pelvino de ovinos: Estudo experimental. Revista Da Faculdade de Zootecnia, Veterinaria e Agronomia, 1(1), 5-10.

Spadeto Junior, O., Rodrigues, L. B., Carvalho, W. T. V., Moreira, D. O., Marval, C. A., Costa, C. G., Alves, G. E. S., Casas, E. B., \& Faleiros, R. R. (2011). Sistemas osso-implante ex vivo utilizando haste intramedular polimérica para imobilização de fraturas femorais em bovinos jovens. Ciência Rural, 41(2), 301-306. DOI: https://doi.org/10.1590/s0103-84782011000200020

Recebido: 18 de fevereiro, 2020

Aprovado: 24 de março, 2010.

Disponível online: 31 julho, 2020.

Licenciamento: Este artigo é publicado na modalidade Acesso Aberto sob a licença Creative Commons Atribuição 4.0 (CC-BY 4.0), a qual permite uso irrestrito, distribuição, reprodução em qualquer meio, desde que o autor e a fonte sejam devidamente creditados. 